\title{
The rate of in vitro fludarabine-induced peripheral blood and bone marrow cell apoptosis may predict the chemotherapy outcome in patients with chronic lymphocytic leukemia
}

\author{
Monika Podhorecka $^{1}$ - Piotr Klimek ${ }^{1}$ - Sylwia Chocholska ${ }^{1}$ - Agnieszka Szymczyk ${ }^{1}$. \\ Arkadiusz Macheta $^{1}$ • Malgorzata Kowal $^{1}$ • Anna Dmoszynska ${ }^{1}$ - Marek Hus ${ }^{1}$
}

Received: 10 March 2015 / Accepted: 23 June 2015 /Published online: 5 July 2015

(C) The Author(s) 2015. This article is published with open access at Springerlink.com

\begin{abstract}
Purpose The problem of drug sensitivity and predicting the outcome of chemotherapy seems to be of great importance in hemato-oncological disorders. There are some factors that can help to predict effects of chemotherapy in chronic lymphocytic leukemia (CLL), such as presence of del17p, del11q, or TP53 gene mutations, which result in resistance to purine analogues and alkylating drugs. Despite the new therapeutic options introduced recently, purine analogues in combination with cyclophosphamide and the monoclonal antibody rituximab is still the gold standard for the first-line treatment of fit patients with CLL. The aim of this study was to assess whether the rate of apoptosis caused by one of purine analoguesfludarabine in cell cultures differs between patients who clinically respond to fludarabine-based chemotherapy and those who do not respond.

Methods CLL leukemic cells, obtained from peripheral blood and bone marrow of 23 patients, were cultured in the presence of fludarabine. After $24 \mathrm{~h}$ of incubation, the rate of apoptosis, indicated by the expression of active caspase-3, was assessed with flow cytometry and then analyzed regarding clinical response to fludarabine-based regimens.

Results The percentage of apoptotic cells induced by fludarabine was significantly higher in the group of patients who achieved remission in comparison to the group with no
\end{abstract}

Electronic supplementary material The online version of this article (doi:10.1007/s00228-015-1893-0) contains supplementary material, which is available to authorized users.

Monika Podhorecka

monika.podhorecka@onet.pl

1 Department of Haematooncology and Bone Marrow Transplantation, Medical University of Lublin, Staszica 11, 20-081 Lublin, Poland response to purine analogues therapy. Interestingly, we observed that among the patients who did not respond to chemotherapy, the presence of del17p and del11q was detected only once. Other non-responders had no detectable genetic abnormalities.

Conclusions Based on these results, it can be presumed that in vitro drug sensitivity test, which is easy to perform, may predict the outcome of fludarabine-based chemotherapy in CLL patients.

Keywords CLL $\cdot$ Apoptosis $\cdot$ Cell culture $\cdot$ Fludarabine

\section{Introduction}

Chronic lymphocytic leukemia (CLL) is characterized by the accumulation of malignant CD19+/CD5+ B cells in blood, bone marrow, and lymphoid organs [1-3]. The leukemic transformation is initiated by genomic alterations causing the deletion of specific micro-RNA genes and increasing the resistance of B cells towards apoptosis [1, 4]. The biology of CLL is also directly entwined with its microenvironment, in which accessory cells can promote leukemia cell growth and survival. Recently, much attention has been focused on the B cell receptor (BCR) and on chemokine receptors that enable CLL cells to home to lymphoid tissues and to establish the leukemia microenvironment [5]. BCR signaling plays an important pathogenic role, based on structural restrictions of the $\mathrm{BCR}$, and BCR-dependent survival and growth of the malignant $B$ cells. In CLL, ligand-independent and liganddependent BCR signaling has been characterized. They can involve mutations of BCR pathway components or be triggered by antigens present in the tissue microenvironment [6].

CLL typically occurs in elderly patients and has a highly variable clinical course [7]. Some patients have indolent 
disease and never need treatment, but in others, the clinical course is aggressive and soon after diagnosis requires intensive treatment $[8,9]$. This variable clinical course of CLL makes the role of prognostic factors very important, especially for distinguishing the group of patients who require intensive treatment from those who will benefit from milder forms of therapy. A number of markers of prognostic relevance have been identified for CLL. Among these markers, biological risk factors such as the $\mathrm{VH}$ mutation status or its surrogate markers as well as genomic aberrations may have the power to identify the subgroup of patients with poor prognosis from early stage patients $[9,10]$. Among them deletions of the short arm of chromosome $17(\operatorname{del}(17 \mathrm{p}))$ or TP53 gene mutation predict resistance to most available therapies [7].

Recently, substantial strides have been made in the treatment of CLL. The novel class of targeted therapies, such as two oral tyrosine kinase inhibitors, ibrutinib (Bruton's kinase inhibitor) and idelalisib (phosphatidylinositol 3-kinase delta inhibitor), have received approval for the treatment of CLL patients. Another promising drugs are BCL-2 protein antagonists that induce apoptosis in leukemic cells. The new monoclonal antibody — obinutuzumab, has also been recently introduced to the therapy of older patients in combination with chlorambucil. Despite the new therapeutic options, purine analogues in combination with cyclophosphamide and the monoclonal antibody rituximab is still the gold standard for the first-line treatment of fit patients with CLL [11]. Some patients, however, are refractory to purine analogues, especially those with del17p or del11q. The presence of other, not explored yet, factors may be responsible for therapy resistance as well. Thus, exploring the process of drug-induced apoptosis in vitro may be of great importance for predicting the susceptibility of leukemic cells to the drug, especially if it would reflect this susceptibility in vivo.

In this study, we attempted to assess the rate of CLL cell apoptosis caused by one of purine analogues - fludarabine. The experiments were performed in short-term cell cultures of peripheral blood and bone marrow supplemented with this drug. We analyzed the percentage of cells with active caspase3 expression. Then, the apoptosis rate was assessed as a marker predicting the outcome of chemotherapy.

\section{Materials and methods}

\section{Patients}

The study was approved by the local ethical committee. Peripheral blood and bone marrow samples were obtained from 23 patients after informed consent. Diagnosis of CLL was based on a clinical examination and morphological and immunological criteria. The patients were enrolled to the study prior to the onset of therapy with fludarabine-based regimens and were not treated previously with any chemotherapeutic agents.

\section{Cell culture}

Mononuclear cells were isolated by density gradient centrifugation using Lymphoprep (Nycomed, Norway). Then, the cells were cultured in medium consisting of RPMI 1640 with $2 \mathrm{mM}$ L-glutamine, 100 units $/ \mathrm{ml}$ penicillin, $100 \mu \mathrm{g} / \mathrm{ml}$ streptomycin, and $10 \%$ fetal calf serum (FCS) at a final concentration of $2 \times 10^{6}$ cells $/ \mathrm{ml}$. This culture medium was supplemented with fludarabine (Schering AG, Germany) at a concentration of $1 \mu \mathrm{g} / \mathrm{ml}$. We selected such a concentration of fludarabine on the basis of our preliminary experiments in CLL cultures, in which this concentration induced significant number of apoptosis with spontaneous apoptosis that did not exceed $50 \%$ of the total cell culture [12]. The cells were cultured at $37{ }^{\circ} \mathrm{C}$ in a $5 \% \mathrm{CO}_{2}$ atmosphere, and they were exposed to the drugs for $24 \mathrm{~h}$. Respective cell samples incubated in the absence of any drug for periods of time equivalent to the drug-treated cells were used as a negative control.

\section{Immunocytochemical detection of active caspase-3 as a marker of apoptosis}

The blood and bone marrow samples that had been treated in culture with fludarabine $\left(\sim 10^{5}\right.$ cells) were initially incubated for 15 min with anti-CD19 PerCP and anti-CD5 APC-conjugated monoclonal antibody (MoAb) (DAKO, Denmark) at room temperature. After fixation and permeabilization procedures (IntraPrep kit, Immunotech, France), the cells were incubated with anti-active caspase-3 MoAb, PE conjugated (Pharmingen, USA), or an isotype-matched negative control (Dako, Denmark) in darkness at room temperature for $15 \mathrm{~min}$. After washing, the labelled cells were analyzed by multiparameter flow cytometry.

\section{Fluorescence measurement}

The samples were measured with a FACSCalibur (Becton Dickinson) using standard emission filters for green and red fluorescence and CellQuest software. The CD5+/CD19+ population was initially gated and further analysis was carried out for this population. Ten thousand cells were measured per sample. To determine the frequency of apoptosis, the percentage of active caspase- 3 cells, corresponding to that in the control cells, was calculated. Intra-assay variation of the flow cytometry test was assessed using two acquisitions per assay, while inter-assay variation was assessed by performing in the chosen cases two cell cultures of the same patient on different days. Both intra- and inter-assay variation were lower than $10 \%$. Representative dot plots illustrating the expression of 
caspase- 3 by CD19+/CD5+ CLL cells in cultures with fludarabine are presented in Fig. 1.

\section{Flow cytometric detection of ZAP-70 and CD38 as prognostic factors}

A total of $1 \times 10^{6}$ peripheral blood cells were stained with the monoclonal antibodies CD19 PE (BD Pharmingen), CD5 CyChrome (Caltag Laboratories, USA), or CD3 PE (BD Pharmingen). Following membrane staining, the cells were fixed in $1 \%$ paraformaldehyde solution in PBS for $15 \mathrm{~min}$ at room temperature and permeabilized with $70 \%$ ethanol for one hour at $-20{ }^{\circ} \mathrm{C}$. After washing with PBS, anti-ZAP-70 antibody (Biomol Research Laboratories, USA) labelled by the ZenonTM Alexa Fluor ${ }^{\circledR} 488$ Mouse IgG2a Labeling Kit (Molecular Probes, USA) was added to the sample tubes. The samples were incubated with the reagent for $30 \mathrm{~min}$ at room temperature, washed once with PBS, and analyzed by flow cytometry (FACSCalibur, Becton Dickinson).

To assess CD38 expression, peripheral blood mononuclear cells were stained with CD38 FITC, CD19 PE, CD5 CyChrome, or IgG1 isotypic control MoAbs. The cells were incubated for $20 \mathrm{~min}$ at room temperature. Finally, the cells were washed and analyzed by flow cytometry.

\section{Fluorescence in situ hybridization}

Fluorescence in situ hybridization (FISH) method was used for an analysis of chromosome alterations characteristic of CLL that are relevant markers of prognosis. The locus-specific probes for 11q22.3 (LSI ATM), 17p13.1 (LSI TP53), 13q.14.3 (D13S319), and the chromosome 12 centromere (CEP12) (Abbott Diagnostics) were used and FISH was performed according to the manufacturer's instructions. Probes were denatured at $73{ }^{\circ} \mathrm{C}$ for $5 \mathrm{~min}$ and then applied to the determined areas on the slides. Following overnight hybridization at $37{ }^{\circ} \mathrm{C}$, the slides were washed and air-dried in the darkness. Then, the slides were stained with DAPI and stored at $-20{ }^{\circ} \mathrm{C}$ in the darkness. The samples were analyzed using the BX51 fluorescence microscope (OLYMPUS) and the CytoVision image analyzing system. At least 200 nuclei were analyzed for each probe with the cutoff value of $20 \%$.
Fig. 1 The representative flow cytometry dot plots showing expression of caspase- 3 by CD19+/CD5+ cells of peripheral blood in CLL patients that were induced to undergo apoptosis by fludarabine. a Patient of responder group with response to fludarabine-based chemiotherapy in vivo. b Patient of nonresponder group with no response to fludarabine-based chemotherapy in vivo
A

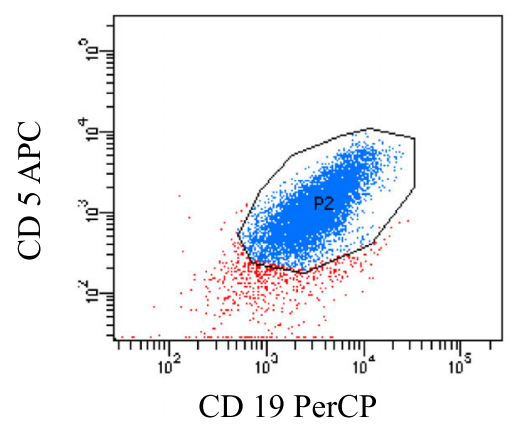

Fludarabine

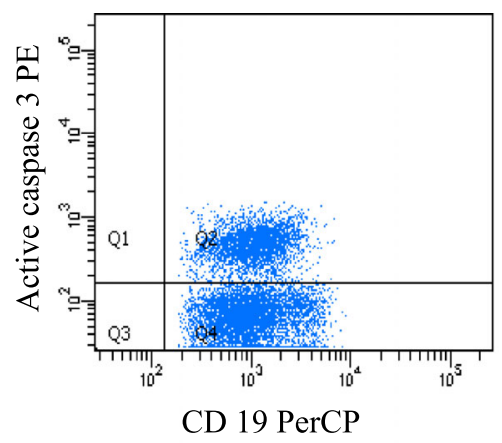

CD 19 PerCP

Responder group

B

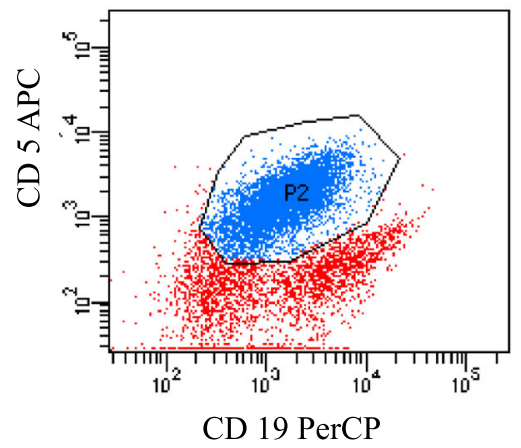

Fludarabine

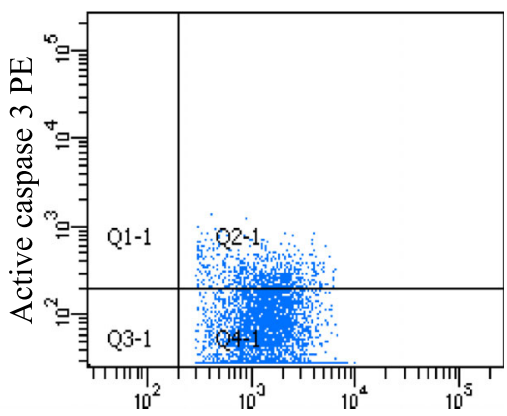

CD 19 PerCP

Non - Responder group 


\section{Clinical response to fludarabine-based treatment regimens}

The assessment of clinical response to chemotherapy with fludarabine-based regimens was performed in the studied group of patients. We used the criteria of response to treatment proposed by WG-IWCLL in 2008 [13] based on WG-NCI criteria from 1996 [14]. According to these criteria, complete response requires the absence of symptoms and organomegaly, normal complete cell counts of peripheral blood, and less than $30 \%$ of lymphocytes in bone marrow for at least 2 months. When size of the lymph nodes, spleen and liver, together with the peripheral blood data, were at least $50 \%$ better than pre-treatment values, the partial response was achieved. Other patients were considered non-responders.

\section{Statistical analysis}

The statistical analysis was performed using STATISTICA 10.0 software for Windows. We used Mann-Whitney $U$ test for two-independent-group analyses. $p<0.05$ was considered to be statistically significant.

\section{Results}

\section{Active caspase-3 expression by CD19+/CD5+ peripheral blood and bone marrow cells in cultures with fludarabine}

We detected active caspase-3 expression in malignant CD19+/ CD5+ cells in all cell cultures from both peripheral blood and bone marrow after $24 \mathrm{~h}$, similarly to our previously published results [12]. An increase in the percentage of caspase-3positive cells was observed after $24 \mathrm{~h}$ in control cultures (cells without drugs) relative to the 0 -h baseline control. This phenomenon was caused by spontaneous apoptosis of malignant cells in in vitro conditions, as it has been reported for CLL [15]. In cell cultures with fludarabine, the percentage of apoptotic cells after $24 \mathrm{~h}$ was obviously higher than at $0 \mathrm{~h}$. It was also significantly higher than the percentage of apoptotic cells in control cultures without drugs after $24 \mathrm{~h}$. The results (presented as the mean \pm standard deviation) are shown in the Table 1 attached as a supplementary document.

\section{Assessment of chemotherapy response}

The patients enrolled into the study were treated with the following fludarabine-based regimens:

- $\quad$ FC (fludarabine + cyclophosphamide), 11 persons

- TF (thalidomide + fludarabine), 5 persons

- FCR (fludarabine + cyclophosphamide + rituximab), 6 persons
- FCA (fludarabine + cyclophosphamide + alemtuzumab), 1 person

For all patients, these regimens were first-line treatment. Thirteen patients achieved complete remission, five partial remission, while five had no treatment response, what was an indication to change the treatment.

\section{Comparison of apoptosis rate in in vitro cultures with response to fludarabine-based chemotherapy}

To assess the rate of apoptosis induced by fludarabine in the group of patients with clinical response to treatment and in the group of non-responders, we measured the expression of active caspase-3 in CD19+/CD5+ peripheral blood and bone marrow samples. Figure 2 illustrates the drug-induced increase in the frequency of apoptotic cells above the level of spontaneous apoptosis seen in the untreated 24-h parallel control cultures in both analyzed groups. The frequency of fludarabine-induced apoptosis in peripheral blood cultures of responding group was significantly higher than in non-responding one, and the same was true when compared with bone marrow cultures. Interestingly, among the patients who did not respond to chemotherapy, only in one case the presence of del17p and in one case del $11 \mathrm{q}$ were detected, while other persons had no genetic abnormalities. Similarly, only two persons were ZAP-70 positive and two were $\mathrm{CD} 38+$. The characteristic of non-responding patients is shown in Table 1.

\section{Discussion}

Purine analogues are currently widely used in CLL patients. Three of them, fludarabine, pentostatin, and cladribine (2CdA), were proved to have an effect on leukemic cells. They demonstrate antitumor activity in some ways such as direct interference with DNA and RNA synthesis, DNA repair mechanisms, induction of apoptosis, control of the cell cycle, and signal transduction pathways [16, 17]. Fludarabine remains the best studied compound of these three in CLL patients. Purine analogues have been reported to induce higher remission rates when they are employed as a first-line therapy compared with other treatment regimens containing alkylating agents or corticosteroids [18-21]. A major advance in CLL treatment was achieved by the combined use of different drugs. Thus, fludarabine-based therapies, in particular, its combination with other drugs such as cyclophosphamide or monoclonal antibody rituximab, were investigated in clinical trials $[22,23]$. It has been proved that patients receiving fludarabine and rituximab had a better progression-free survival and overall survival than patients receiving fludarabine alone that represent a significant advance in CLL therapy [24, 25]. 

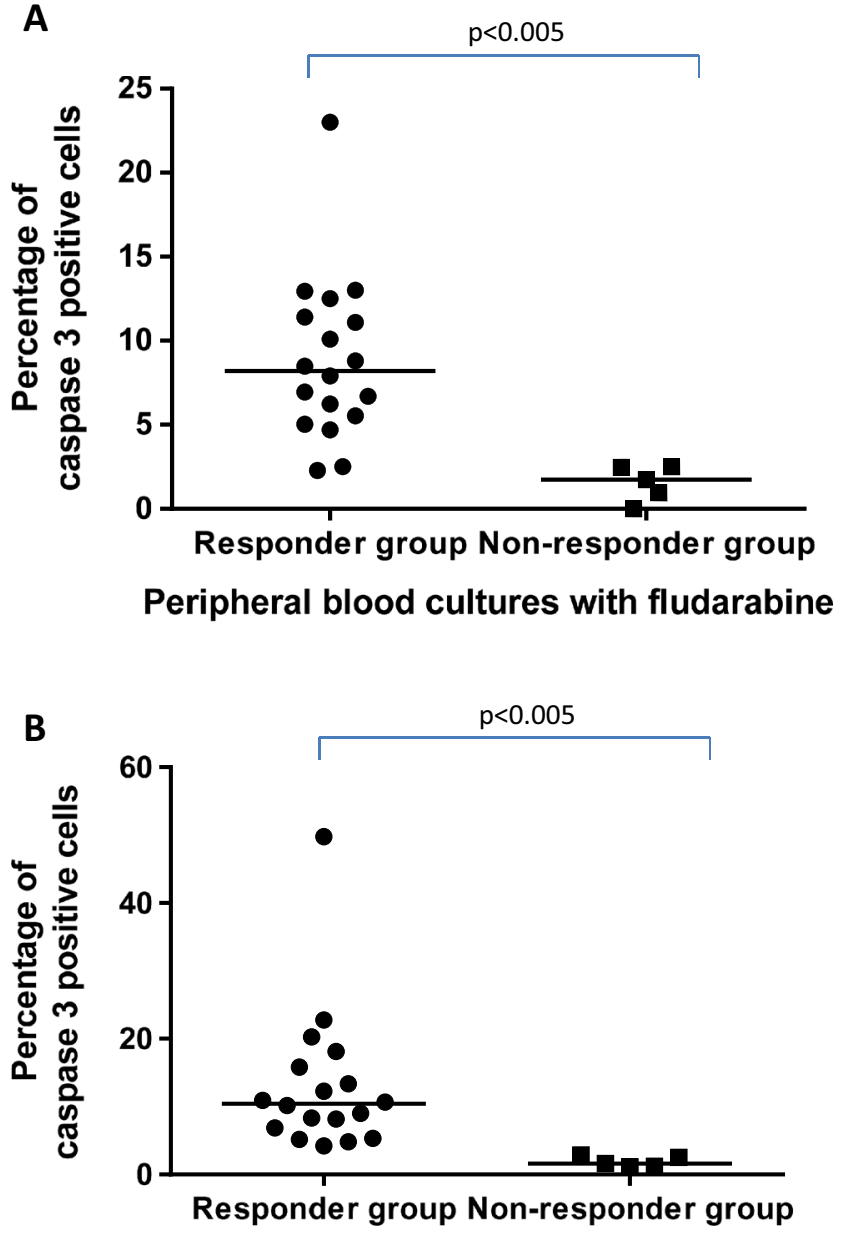

Bone marrow cultures with fludarabine

Fig. 2 Percentage of caspase-3-positive leukemic CD19+/CD5+ cells in 24-h fludarabine-induced cell cultures in group of patients who clinically respond to fludarabine-based chemotherapy $(n=18)$ versus group of nonresponders $(n=5)$. Data represent the drug-induced increase in the percentage of apoptotic cells above the respective values observed in control cultures of the same cell population. Statistically significant differences are indicated. a Peripheral blood samples. b Bone marrow samples

A number of markers of prognostic relevance have been identified for CLL patients, such as laboratory parameters reflecting the tumor burden or disease activity and markers related to the biology of the leukemia. The latter include genetic markers (like genomic aberrations, gene abnormalities (p53 and ATM), and the mutation status of the variable segment of immunoglobulin heavy chain genes $(\operatorname{IgVH}))$ or surrogate markers for these factors such as ZAP-70 or CD38 $[9,25,26]$. Some of the prognostic factors can be used as predictors of the outcome of chemotherapy. Deletions of the short arm of chromosome 17 (del17p) are found in 5-8 \% of chemotherapy-resistant patients. These deletions almost always include band $17 \mathrm{p} 13$, where the prominent tumor suppressor gene TP53 is located. CLL patients carrying a del17p clone show marked resistance to genotoxic chemotherapies that cannot be overcome by the addition of anti-CD20 antibodies [7, 27]. Mutations of TP53 are associated with very poor prognosis and are found in 4-37\% of patients with CLL [28]. The majority of patients with cytogenetically detected del17p show mutations in the remaining TP53 allele. In cases without del17p, TP53 mutations are much rarer, but have a similarly detrimental effect on chemotherapy response and overall survival [29]. However, the assessment of TP53 mutation needs complicated and time-consuming methods, not widely available.

In the presented study, we tried to assess whether the rate of apoptosis caused by fludarabine in vitro can predict the outcome of chemotherapy in vivo. The experiments were performed in cultures of blood and bone marrow cells obtained from CLL patients prior to the onset of fludarabine-based treatment. The cultures were supplemented with fludarabine, and the percentage of caspase-3-positive cells after a 24-h period was determined. The results of the study showed statistically significant differences in apoptosis rate between the group of responders and non-responders as far as clinical response was concerned. The percentage of apoptotic cells was higher both in peripheral blood and bone marrow samples in the group of patients who obtained clinically complete or partial remission in comparison to the group with no response to fludarabine therapy. Interestingly, we observed that among the patients who did not respond to chemotherapy only in one case the presence of del17p and in one case del11q predicting purine analogues resistance, were detected. Other persons of this group had no genetic abnormalities. Nevertheless, it is true that we did not analyze the TP53 mutations in the group of our patients. Such an analysis is elaborate, time-consuming, and impossible to perform in many hospitals. On the basis of our results, it can be concluded that quite easy to perform and simple assessment of apoptosis in drug-supplemented cultures may be also useful to predict treatment results. Furthermore, in
Table 1 Clinical characteristic of patients who did not respond to the therapy based on fludarabine regimens

\begin{tabular}{lllll}
\hline Patient number & Rai stadium & ZAP-70 & CD38 & Cytogenetic characteristic \\
\hline 1 & 4 & - & - & No changes \\
2 & 4 & + & + & No changes \\
3 & 2 & + & + & del17p \\
4 & 2 & - & - & No changes \\
5 & 1 & - & - & del11q \\
\hline
\end{tabular}


our study, only two persons of the non-responders group expressed worse prognostic markers such as ZAP-70 and CD38, which indicates usefulness of the apoptosis test in predicting the outcome of therapy even in patients without expression of worse prognostic markers.

The seminal work in the field of in vitro prediction of clinical outcome in CLL was reported by Bosanquet et al. [30-32]. The authors used the differential staining cytotoxicity (DiSC) assay, as ex vivo apoptotic drug response test, to identify the sensitivity or resistance to fludarabine of lymphocytes from CLL patients. The results were compared with subsequent patient treatment, response, and survival. Treating fludarabine-test-resistant patients with fludarabine in vivo resulted in poor response and short survival compared with fludarabine-test-sensitive patients [30]. It was shown that fludarabine-test-resistance by DiSC assay is a powerful independent prognostic factor for CLL patients, which was in accordance with our results. The authors concluded that the DiSC assay could be considered a cost-effective guide to the treatment of chronic lymphocytic leukemia [31, 32].

Bromidge et al. [33] assessed the in vitro sensitivity of CLL cells to purine analogues and its correlation with clinical course in the group of 51 persons. Their results showed that the rate of apoptosis in cell cultures was comparable between group of patients who needed treatment and the group with stable disease. For this reason, such a test cannot be used to predict the time to start the therapy. Similarly to our study, the authors showed that sensitivity of leukemic cells to in vitro induced apoptosis correlated with clinical effects of therapy. Castejon et al. [34] analyzed fludarabine- and cladribineinduced apoptosis of CLL cells in vitro in comparison to in vivo response to chemotherapy with these drugs. The analysis was performed in the group of 50 CLL patients. Similarly to our results, in vitro apoptosis was in significant correlation with clinical response to treatment.

Żołnierczyk et al. [35] analyzed ex vivo sensitivity of leukemic cells obtained from CLL patients to conventional purine analogues and the selective CDK inhibitor R-roscovitine with and without the addition of an alkylating agent, prior to the onset of in vivo therapy. The kinetics and rate of spontaneous and drug-induced apoptosis of CLL cells under ex vivo conditions differed significantly between patients, reflecting the variability observed during in vivo treatment. Similarly to our results, the authors concluded that ex vivo testing might be useful for identifying the most potent first-line therapeutic regimen for specific CLL patients and possibly for the design of therapies tailored for individual CLL patients [35]. We showed additionally that sensitivity of leukemic cells to purine analogues in vitro seemed to be independent of cytogenetic changes detected in cells.

Recently, it has been reported that not only drug-induced apoptosis but also spontaneous programmed death may be a simple prognostic test which can predict the course of the disease and response to treatment. Witkowska et al. [36] compared the level of spontaneous apoptosis with prognostic factors and clinical course of the disease in 135 treatment naive patients with CLL. Rate of apoptosis in patients with stable disease was found to be significantly higher than in the group with progressive course of the disease. Furthermore, the level of apoptosis correlated inversely with the progression-free survival.

In conclusion, the obtained results indicate that the sensitivity of CLL cells to fludarabine induced apoptosis in in vitro conditions may reflect the effects of these drugs in vivo. Assessment of drug-induced apoptosis in cell cultures seems to be an easy to perform and simple test predicting the outcome of chemotherapy with fludarabine CLL patients.

Authors' contributions MP designed the study, contributed to the data collection and analysis, and wrote the manuscript. PK, SCh, ASz, AM, and MK contributed to the data collection and analysis. AD and $\mathrm{MH}$ contributed to the interpretation of the data and critical revision of the manuscript for important intellectual content.

Conflict of interest The authors declare that they have no competing interest.

Open Access This article is distributed under the terms of the Creative Commons Attribution 4.0 International License (http://creativecommons.org/licenses/by/4.0/), which permits unrestricted use, distribution, and reproduction in any medium, provided you give appropriate credit to the original author(s) and the source, provide a link to the Creative Commons license, and indicate if changes were made.

\section{References}

1. Calligaris-Cappio F, Hamblin TJ (1999) B-cell chronic lymphocytic leukemia: a bird of a different feather. J Clin Oncol 17:399-408

2. Chiorazzi N, Rai KR, Ferrarini M (2005) Chronic lymphocytic leukemia. N Engl J Med 352:804-815

3. Hamblin TJ, Oscier DG (1997) Chronic lymphocytic leukemia: the nature of the leukemic cells. Blood Rev 11:119-122

4. Calin GA, Dumitru CD, Shimizu M et al (2002) Frequent deletions and downregulation of micro-RNA genes miR15 and miR16 at $13 q 14$ in chronic lymphocytic leukemia. Proc Natl Acad Sci U S A 99:15524-15529

5. Zhang S, Kipps TJ (2014) The pathogenesis of chronic lymphocytic leukemia. Annu Rev Pathol 9:103-118

6. Burger JA, Nicholas Chiorazzi N (2013) B cell receptor signaling in chronic lymphocytic leukemia. Trends Immunol 34:592-601

7. Hallek M (2013) Chronic lymphocytic leukemia: 2013 update on diagnosis, risk stratification and treatment. Am J Hematol 88:804 816

8. Messmer BT, Messmer D, Allen SL et al (2005) In vivo measurements document the dynamic cellular kinetics of chronic lymphocytic leukemia B cells. J Clin Invest 115:755-764

9. Stilgenbauer S (2006) Chronic lymphocytic leukemia: genetics for predicting outcome. Hematology 2:185-190 
10. Gribben JG (2008) Molecular profiling in CLL. Hematology Am Soc Hematol Educ Program 1:444-449

11. Tausch E, Mertens D, Stilgenbauer S (2014) Advances in treating chronic lymphocytic leukemia. F1000Prime Rep 6:65-72

12. Podhorecka M, Klimek P, Kowal M, Chocholska S, Bojarska-Junak A, Dmoszynska A (2010) Assessment of peripheral blood and bone marrow cells apoptosis caused by purine analogues in patients with chronic lymphocytic leukemia in correlation with parameters of disease progression. Acta Haematol 123:171-178

13. Hallek M, Cheson BD, Catovsky D et al (2008) International workshop on chronic lymphocytic leukemia. Guidelines for the diagnosis and treatment of chronic lymphocytic leukemia: a report from the International Workshop on Chronic Lymphocytic Leukemia updating the National Cancer Institute-Working Group 1996 guidelines. Blood 111:5446-5456

14. Cheson BD, Bennett JM, Grever M et al (1996) National Cancer Institute-sponsored Working Group guidelines for chronic lymphocytic leukemia: revised guidelines for diagnosis and treatment. Blood 87:4990-4997

15. Collins RJ, Verschuer LA, Harmon BY, Prentice RL, Pope JH, Kerr JF (1989) Spontaneous programmed death (apoptosis) of B-chronic lymphocytic leukemia cells following their culture in vitro. Br J Haematol 71:343-350

16. Kobylinska A, Bednarek J, Blonski JZ, Hanausek M, Walaszek Z, Robak T, Kilianska ZM (2006) In vitro sensitivity of B-cell chronic lymphocytic leukemia to cladribine and its combinations with mafosfamide and/or mitoxantrone. Oncol Rep 16:1389-1395

17. Robak T (2005) Therapy of chronic lymphocytic leukemia with purine analogs and monoclonal antibodies. Transfus Apher Sci 32:33-44

18. Rai KR, Peterson BL, Appelbaum FR et al (2000) Fludarabine compared with chlorambucil as primary therapy for chronic lymphocytic leukemia. N Engl J Med 343:1750-1757

19. Nabhan C, Gartenhaus RB, Tallman MS (2004) Purine nucleoside analogues and combination therapies in B-cell chronic lymphocytic leukemia: dawn of a new era. Leuk Res 28:429-442

20. Van den Neste E, Cardoen S, Offner F, Bontemps F (2005) Old and new insights into the mechanisms of action of two nucleoside analogs active in lymphoid malignancies: fludarabine and cladribine (review). Int J Oncol 27:1113-1124

21. Leporrier M, Chevret S, Cazin B et al (2001) Randomized comparison of fludarabine, CAP, and ChOP in 938 previously untreated stage $\mathrm{B}$ and $\mathrm{C}$ chronic lymphocytic leukemia patients. Blood 98: 2319-2325

22. Hallek M, Eichhorst BF (2004) Chemotherapy combination treatment regimens with fludarabine in chronic lymphocytic leukemia. Hematol J 5:20-30

23. Catovsky D, Richards S, Matutes E et al (2007) Assessment of fludarabine plus cyclophosphamide for patients with chronic lymphocytic leukaemia (the LRF CLL4 Trial): a randomised controlled trial. Lancet 370:230-239
24. Byrd JC, Rai K, Peterson BL et al (2005) Addition of rituximab to fludarabine may prolong progression-free survival and overall survival in patients with previously untreated chronic lymphocytic leukemia: an updated retrospective comparative analysis of CALGB 9712 and CALGB 9011. Blood 105:49-53

25. Hamblin TJ, Davis Z, Gardiner A, Oscier DG, Stevenson FK (1999) Unmutated Ig V(H) genes are associated with a more aggressive form of chronic lymphocytic leukemia. Blood 94:18481854

26. Crespo M, Bosch F, Villamor N et al (2003) ZAP-70 expression as a surrogate for immunoglobulin-variable-region mutations in chronic lymphocytic leukemia. N Engl J Med 348:1764-1775

27. Hallek M, Fischer K, Fingerle-Rowson G et al (2010) Addition of rituximab to fludarabine and cyclophosphamide in patients with chronic lymphocytic leukaemia: a randomised, open-label, phase 3 trial. Lancet 376:1164-1174

28. Zenz T, Vollmer D, Trbusek M et al (2010) TP53 mutation profile in chronic lymphocytic leukemia: evidence for a disease specific profile from a comprehensive analysis of 268 mutations. Leukemia 24: 2072-2079

29. Seiffert M, Dietrich S, Jethwa A et al (2012) Exploiting biological diversity and genomic aberrations in chronic lymphocytic leukemia. Leuk Lymphoma 53:1023-1031

30. Bosanquet AG, Johnson SA, Richards SM (1999) Prognosis for fludarabine therapy of chronic lymphocytic leukaemia based on ex vivo drug response by DiSC assay. Br J Haematol 106:71-77

31. Bosanquet AG (1991) Correlations between therapeutic response of leukaemias and in-vitro drug-sensitivity assay. Lancet 337:711-714

32. Mason JM, Drummond MF, Bosanquet AG, Sheldon TA (1999) The DiSC assay. A cost-effective guide to treatment for chronic lymphocytic leukemia? Int J Technol Assess Health Care 15:173184

33. Bromidge TJ, Turner DL, Howe DJ, Johnson SA, Rule SA (1998) In vitro chemosensitivity of chronic lymphocytic leukaemia to purine analogues - correlation with clinical course. Leukemia 12: $1230-1235$

34. Castejón R, Yebra M, Citores MJ, Villarreal M, García-Marco JA, Vargas JA (2009) Drug induction apoptosis assay as predictive value of chemotherapy response in patients with B-cell chronic lymphocytic leukemia. Leuk Lymphoma 50:593-603

35. Żołnierczyk JD, Komina O, Błoński JZ, Borowiak A, CebulaObrzut B, Smolewski P, Robak P, Kiliańska ZM, WęsierskaGadek J (2012) Can ex vivo evaluation (testing) predict the sensitivity of CLL cells to therapy with purine analogs in conjunction with an alkylating agent? A comparison of in vivo and ex vivo responses to treatment. Med Oncol 29:2111-2126

36. Witkowska M, Nowak W, Cebula-Obrzut B, Majchrzak A, Medra A, Robak T, Smolewski P (2014) Spontaneous in vitro apoptosis of de novo chronic lymphocytic leukemia cells correlates with risk of the disease progression. Cytometry B Clin Cytom 86:410-417 